\title{
ANIMATING MULTIPLE INTERACTING MISCIBLE AND IMMISCIBLE FLUIDS BASED ON PARTICLE SIMULATION
}

\author{
JURAJ ONDERIK, MICHAL CHLÁDEK AND ROMAN ĎURIKOVIČ
}

\begin{abstract}
We present a particle-based approach for animating multiple interacting liquids that can handle number of immiscible fluids as well as number of miscible fluids in our simulation framework. We solve the usual problem of robust interface tracking, between immiscible fluids, by reconstructing the zero level set of our novel composite implicit function, see Fig. 1 left and center. It's recurrent formulation handles directly interfaces between any number of liquids including the free surfaces. We model the miscible fluids by tracking concentrations of dissolved materials in the vicinity of each particle. Flick's law is applied for the Laplacian-based diffusion of concentrations, see Fig. 1 right. Particle sedimentation is achieved by directional advection along the settling velocity. The diffusion-advection equation is discretized by particles using the Lagrangian formulation. The proposed improvements can be easily implemented into the common Smoothed Particle Hydrodynamics (SPH) simulations framework.
\end{abstract}

Mathematics Subject Classification 2000: I.3.5, I.3.7

Additional Key Words and Phrases: Multi-fluid flow, Miscible fluid, Immiscible fluid, SPH particlebased simulation
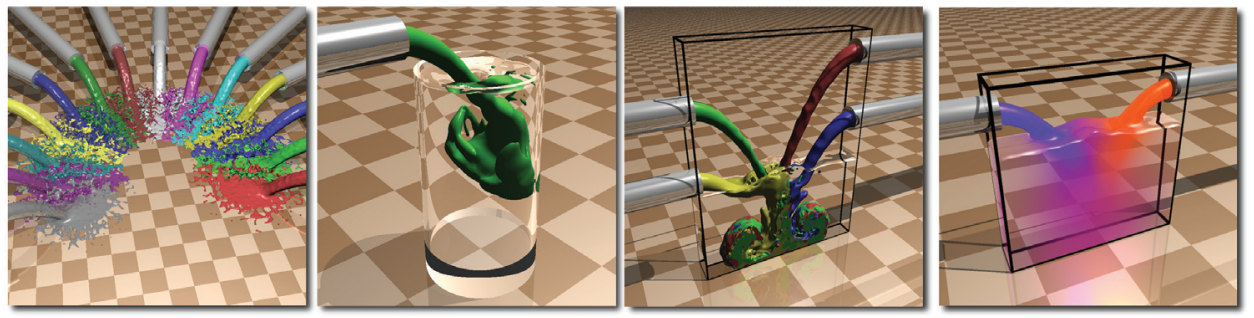

Fig. 1. Examples of mixing fluids from left to right: Mixing of 14 different immiscible fluids. Pouring a green fluid immiscible with water into glass of water. Poring two different miscible fluids into the aquarium.

\section{INTRODUCTION}

A number of great works have been published in the recent years focusing on simulation of single or two-phase fluids. Only a few other (eg. [Losasso et al. 2006; Kim 2010]) addressed the simulation and surface reconstruction of multiple fluids. Although mixing of 
several fluids is not unusual in the real world little attention have been paid to the simulation of multiple interacting fluids. In contrast to the single-phase scenario, multiple-phase simulations pose several interesting challenges ranging from immiscible to miscible problems.

Unique and consistent definition of interfaces between immiscible fluids is still an issue especially in particle-based simulations. Methods proposed for single-fluid scenarios usually fail on the common interfaces. Gaps and overlaps emerge near the interface. In this paper, we propose a novel definition of a composite iso-surface which provides a unique definition of the common interface. Using this definition we are able to track interfaces as well as free surfaces between multiple interacting fluids.

A straightforward way to simulation of miscible fluids is adding of diffusion forces to the simulation. The drawback of such solution is the need of a large resolution of the fluid in order to achieve visually plausible results for the purpose of computer graphics. Therefore we exploit a concentration based approach, where the concentration of each fluid is tracked at the particles and advected in Eulerian way. In this we are able to simulate miscible avoiding large particle number. Adding of directional advection of concentrations enabled us to model simplified deposition of dissolved materials and thus can be used for modeling erosion and sedimentation.
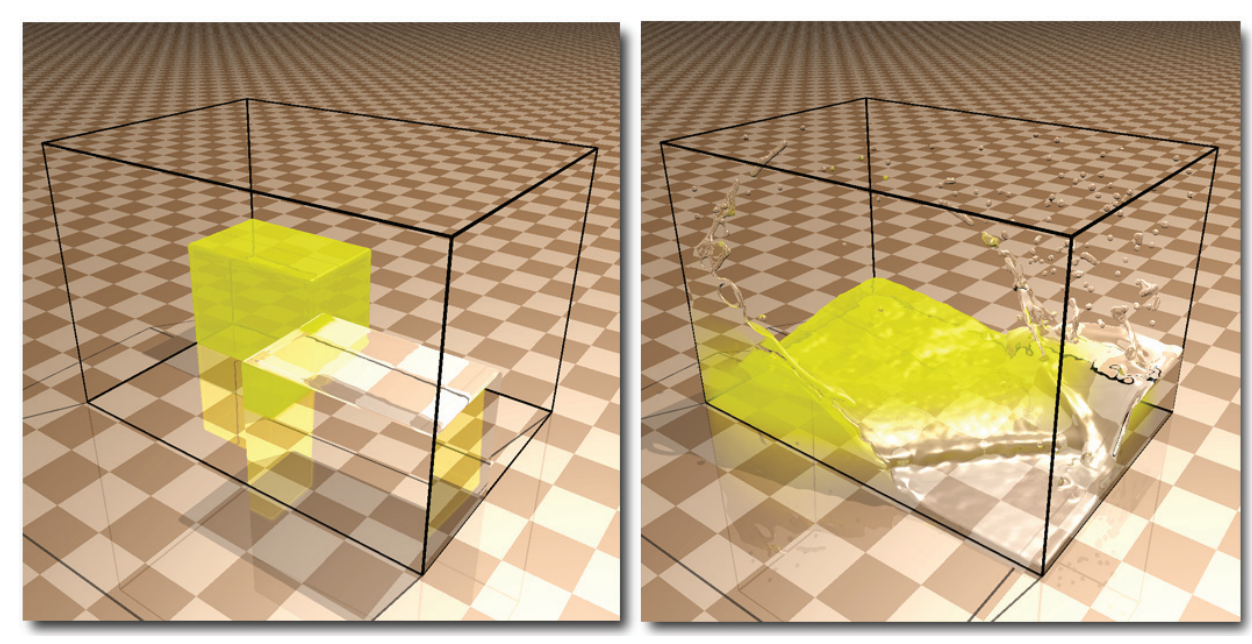

Fig. 2. A dam break scene with two miscible fluids having different viscosities.

Our paper is structured as follows: In Section 2 we discuss the previous work done in this area and in Section 3 we give a brief description of used simulation method. Afterwards, we propose a surface definition for multiple imminscible fluids in Section 5. In Section 6, we explain concentration based approach used for simulation of diffusion and sedimentation. We give the results and discuss our methods in Section 7. 


\section{RELATED WORK}

Within Eulerian simulations, high quality visualizations of interfaces between multiple immiscible fluids were achieved using multiple level sets [Losasso et al. 2006]. Gradient based projection was used to fill vacuum regions and interface overlaps. Recently, regional level sets [Kim 2010] has been used to solve the increasing number of level set variables with each different material. Although an unified particle based models for interaction of solids and fluids has been successfully provided [Solenthaler et al. 2007; Keiser et al. 2005], visualization of interfaces between more than 2 particle-based liquid has not been sufficiently addressed yet. In order to cope with these problems we propose a novel concept of composite implicit function.

Interaction of multiple immiscible fluids has been animated using several projected level sets [Losasso et al. 2006]. Melting and burning effects were achieved using Eulerian simulation in [Losasso et al. 2006; Carlson et al. 2002]. In terms of SPH interaction of water and air with temperature advection and diffusion was presented in [Müller et al. 2005] and later extended into an unified particle based simulation of solids and fluids [Keiser et al. 2005].

Within Eulerian context, a single miscible fluid was first demonstrated to graphics community by modeling smoke diffusion and cloud formation [Stam 1999; Fedkiw et al. 2001; Rasmussen et al. 2003; Harris et al. 2003], where the density and the temperature are advected and diffused. Interaction between multiple miscible fluids with a free surface were studied later in [Kang et al. 2010; Shiny et al. 2010]. Phase Field Method has been used to handle interface problem occurring between miscible and immiscible fluids within a lattice Boltzmann model [Park et al. 2008]. Cahn-Hilliard equation was employed to evolve density fractions representing the amount of each fluid within a cell. Volume of fluid method has been applied to capture interfaces between several miscible fluids with a second order accuracy in [Bao et al. 2010]. Miscibility is achieved by evaluation of an advection-diffusion equation. Recently, advanced phenomena as viscous fingering for miscible fluids has been presented using Darcy's Law within a hybrid grid based and SPH simulation framework [Shiny et al. 2010]. In [Kang et al. 2010] another hybrid approach for miscible and immiscible fluids was presented. Particle level sets are employed to track interfaces between immiscible fluids, while volume fractions are diffusing to model miscible effects. The proposed method correctly handles variable densities, viscosities and material diffusion. The proposed concept uses molecular diffusion and directional advection of concentrations on nearby particles. A Eulerian-based erosion and Lagrangian-based fluid simulation was coupled in [Krištof et al. 2009]. The SPH method was used to simulate the fluid flow. The eroded material was associated with the fluid particles and moved implicitly with particles and explicitly based on an additional velocity field. This field represented the material diffusion in the fluid.

\section{SPH SIMULATION BACKGROUND}

For the fluid simulation, SPH algorithm is used that is a Lagrangian fluid simulation method. Fluid is discretized by particles taken as known samples. Any property A of 


\section{J. ONDERIK, M. CHLÁDEK, R. ĎURIKOVIČ}

the fluid is approximated by summing over neighboring particles in the following way:

$$
A(\mathbf{p}) \approx\langle A\rangle(\mathbf{p})=\sum_{j} \frac{m_{j}}{\rho_{j}} A_{j} W\left(\left\|\mathbf{p}-\mathbf{p}_{j}\right\|, h\right),
$$

where $m_{j}, \rho_{j}, A_{j}$ and $\mathbf{p}_{j}$ is the mass, density, property A and position of particle $j$, respectively. $W$ is a smoothing kernel function with the smoothing length $h$. To compute pressure forces acting in the fluid we use Tait equation proposed in [Batchelor 1967].

The pressure and viscous forces are derived from the Navier-Stokes equations and can have various forms because of the symmetrization. We used the SPH force equations proposed in [Müller et al. 2005] and smoothing kernel functions given in [Müller et al. 2003].

\section{SURFACE RECONSTRUCTION FROM PARTICLES BELONGING TO A SINGLE FLUID}

Compact and smooth surface is essential to obtain visually plausible results of simulated liquids. We have improved the surface reconstruction techniques [Zhu and Bridson 2005; Solenthaler et al. 2007] to handle uneven distributions of particles and propose a novel approach to reduce artifacts due to isolated particles. Our method does not require computationally expensive eigenvalue analysis and gives us a clear expression of the surface normal. We give here a brief overview. Similarly to [Solenthaler et al. 2007], we reduce
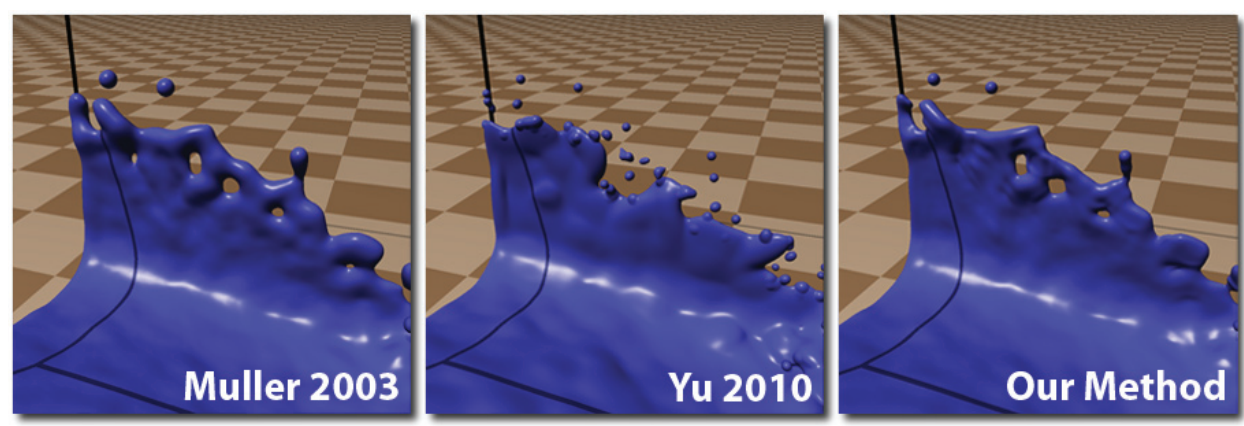

Fig. 3. Left: Thick and bloby approach by [Müller et al. 2003]. Middle: Distance based method of [Zhu and Bridson 2005]. Right: Our density normalized method. Both thick and ghost regions are removed, making thin shells more compact.

"ghost" artifacts of surface reconstruction method proposed in [Zhu and Bridson 2005] by modulating the surface distance $R$ with a decay function $f(\mathbf{p})$

$$
\phi(\mathbf{p})=\|\mathbf{p}-\mathbf{C}(\mathbf{p})\|-R f(\mathbf{p}) .
$$

The central function $\mathbf{C}(\mathbf{p})$ is a weighted average of neighbor particles normalized with iso-density $w_{j}$ :

$$
\mathbf{C}(\mathbf{p})=\frac{\sum_{j} \frac{1}{w_{j}} W\left(\left\|\mathbf{p}-\mathbf{p}_{j}\right\|, h\right) \mathbf{p}_{j}}{\sum_{j} \frac{1}{w_{j}} W\left(\left\|\mathbf{p}-\mathbf{p}_{j}\right\|, h\right)} .
$$


The iso-density $w_{j}$ of $j$-th particle is calculated using SPH approximation of unit mass of neighboring particles

$$
w_{j}=\sum_{i} W\left(\left\|\mathbf{p}_{j}-\mathbf{p}_{i}\right\|, h\right), \quad W(r, h)=\left(1-\frac{r^{2}}{h^{2}}\right)^{3},
$$

where $W(r, h)$ is a polynomial smoothing kernel function. Distance decay function $f$ is a factor that controls how far is the iso-surface from the particles and is defined as a composition of central function $\mathbf{C}$, normalized iso-density $w$ and transfer function $g$ :

$$
\begin{aligned}
& f(\mathbf{p})=g(w(\mathbf{C}(\mathbf{p}))), \\
& w(\mathbf{c})=\sum_{j} \frac{1}{w_{j}} W\left(\left\|\mathbf{c}-\mathbf{p}_{j}\right\|, h\right), \\
& g(w)=\left(1-\frac{\left(w-w_{\max }\right)^{2}}{\left(w_{\max }-w_{\min }\right)^{2}}\right)^{2} .
\end{aligned}
$$

Applying the iso-density normalization $w$ to the central function $C$ and the density based modulation of the surface distance $R$ provides us with a smooth and solid definition of implicit surface reducing "ghost" artifacts even within irregular particles distributions, see Figure 3.

\section{SURFACE RECONSTRUCTION FROM PARTICLES BELONGING TO IMMISCI- BLE FLUIDS}

We propose a general approach of surface reconstruction assuming $K$ different immiscible fluids. We assign a fluid identifier to each particle. For the $k$-th fluid we have an isofunction $\phi_{k}(\mathbf{p})$ and an isovalue $s_{k}$ giving us an implicit function $\phi_{k}(\mathbf{p})-s_{k}=0$. This implicit function defines the fluid surface with respect to the particles of $k$-th fluid only. We have used three different definitions of the function $\phi_{k}(\mathbf{p})$ from [Müller et al. 2003; Yu and Turk 2010; Onderik et al. 2011]. Such approach successfully generates an in-between interface for each fluid, unfortunately they penetrate each other near the common interface.

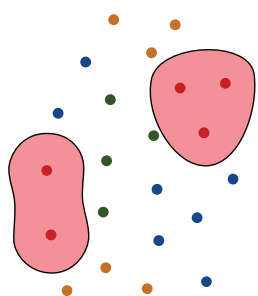

ONE FLUID

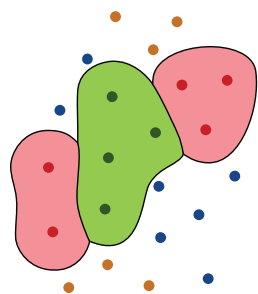

TWO FLUIDS

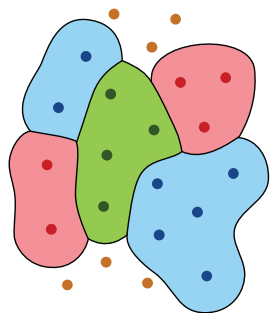

THREE FLUIDS

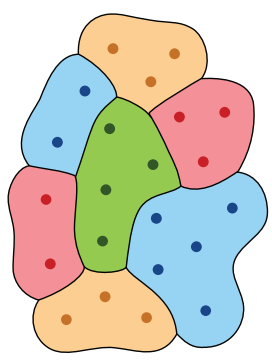

FOUR FLUIDS

Fig. 4. 2D results of our recurrent algorithm constructing a zero level set of the composite iso-potential for 4 immiscible fluids. From left to right, interfaces of 1,2,3 and 4 fluids are generated. Notice several sharp "corners" caused by the absolute value used in the implicit function definition. 
We overcome this drawback by defining a composite implicit surface $\varphi(\mathbf{p})=0$ using the following recurrent formulation

$$
\begin{aligned}
\varphi_{k}(\mathbf{p}) & =\left|\varphi_{k-1}(\mathbf{p})-\max \left(0, \phi_{k}(\mathbf{p})\right)\right|, \\
\varphi_{0}(\mathbf{p}) & =0, \\
\varphi(\mathbf{p}) & =\varphi_{K}(\mathbf{p})-s,
\end{aligned}
$$

where $k=1, \ldots, K, K$ is the number of different fluids and $s$ is the isolevel defining the implicit function. It is worth to note that the definition from Equation 6 is not commutative but during our experiments only very small deterrences were achieved by using different order of combination.

As shown in Figure 4, our composite iso-potential provides a unique zero level implicit surface definition for both the free-surfaces and the fluid interfaces. Using the absolute value in our composite iso-function makes the resulting surface only piece-wise smooth and thus enabling to model the sharp features desired for complex interfaces of multiple immiscible fluids.

Further processing (e.g. ray tracing) of an interface usually requires to evaluate the normal and identify the fluid to which the examined point belongs to. Noticing that the gradient of absolute of real function $f(\mathbf{p})$ is $\nabla|f(\mathbf{p})|=\frac{f(\mathbf{p})}{|f(\mathbf{p})|} \nabla f(\mathbf{p})=\operatorname{sgn}(f(\mathbf{p})) \nabla f(\mathbf{p})$ the surface normal $\mathbf{n}_{p}$ of our composite implicit function $\varphi(\mathbf{p})$ is given as

$$
\begin{aligned}
& \mathbf{n}_{p}=\nabla \varphi(\mathbf{p})=\nabla \varphi_{K}(\mathbf{p}) \\
& \nabla \varphi_{k}(\mathbf{p})=\nabla\left|\varphi_{k-1}(\mathbf{p})-\max \left(0, \phi_{k}(\mathbf{p})\right)\right|= \\
& =\operatorname{sgn}\left(\varphi_{k}(\mathbf{p})\right)\left(\nabla \varphi_{k-1}(\mathbf{p})-\right. \\
& \left.\quad-\operatorname{sgn}\left(\max \left(0, \phi_{k}(\mathbf{p})\right)\right) \nabla \phi_{k}(\mathbf{p})\right) .
\end{aligned}
$$

Assuming we have $K$ fluids identified with numbers $1, \cdots, K$, we can assign the fluid identifier at point $\mathbf{p}$ by the following classification function $F(\mathbf{p})$

$$
\begin{aligned}
& F_{k}(\mathbf{p})=\left\{\begin{array}{ll}
k & 0<\varphi_{k}(\mathbf{p})<\phi_{k}(\mathbf{p}) \\
F_{k-1}(\mathbf{p}) & \text { otherwise }
\end{array},\right. \\
& F(\mathbf{p})=F_{K}(\mathbf{p}),
\end{aligned}
$$

where $k=1, \ldots, K$. Points that are outside of any fluid are identified by $F_{0}(\mathbf{p}):=0$.

\section{MISCIBLE FLUIDS SIMULATION}

In this section we contribute by proposing a generalized strategy for simulating multiple miscible fluids. We propose an approach based on time evolving concentrations using Laplacian diffusion. Such technique can be easily implemented, it is capable of handling any number of fluids and it can be applied to almost any simulated attribute of particles. Adding directional advection of concentrations enables us to model simplified deposition 
of dissolved materials and thus can be used for modeling erosion and sedimentation within particle-based fluids.
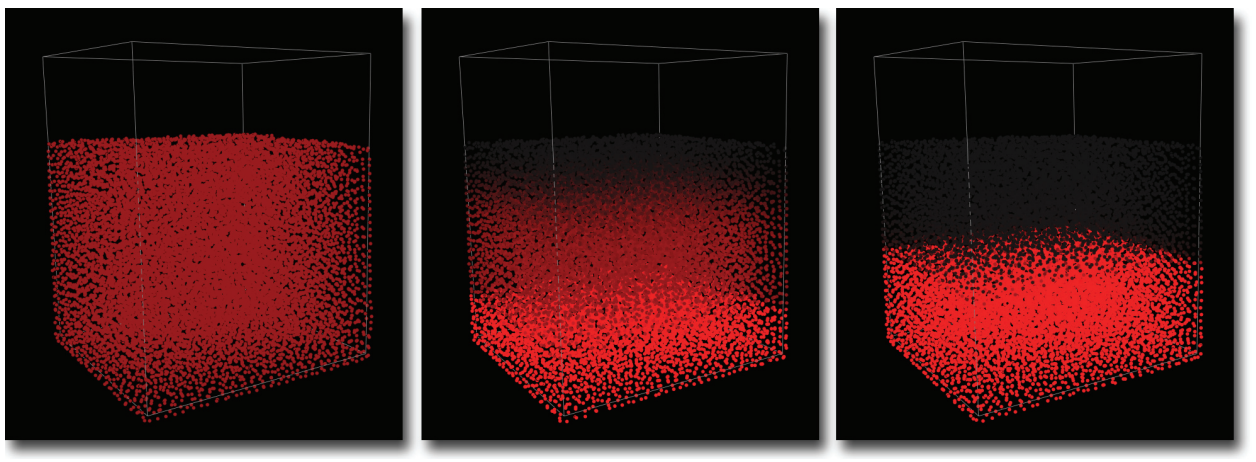

Fig. 5. Simulation of the sedimentation using the particle based settling advection. Initial scene(left) consists of all particles having 50\% concentration of dissolved material (red). At the end of the animation(right), the bottom half of particles is saturated $(100 \%)$, thus the sedimentation stops.

Since material advection is implicitly handled within SPH technique, animation of multiple immiscible fluids can be approximated by simulating many small particles with various material properties. Instead of mixing enormously large number of different small particles to mimic mixtures, we can approximate particles within a reasonable volume with one "mixture" particle having the weighted average of their material properties.

Inspired by particle based simulation of porous materials [Lenaerts et al. 2008; Rungjiratananon et al. 2008] we use concentrations to track the amount of each mixed fluid dissolved within the volume of one mixture particle. Since each mixture particle represents a small finite volume of the fluid the physical behavior depends on material properties of all dissolved fluids in it. When modeling $K$ different miscible fluids, we assign to each mixture particle $\mathbf{p}_{i}$ all $K$ concentrations $C_{i}^{1} \cdots C_{i}^{K}$ and model any miscible physical quantity $A_{i}$ by the following weighted average

$$
A_{i}=\sum_{k} C_{i}^{k} A_{i}^{k}, \quad \text { where } \quad \sum_{k} C_{i}^{k}=1,
$$

and $C_{i}^{k} \in[0,1]$. For example, fluids with particles having different weights, $i$-th mixture particle has the weight $m_{i}=\sum_{k} C_{i}^{k} m^{k}$.

Velocity driven advection of mixture particles, however, does not sufficiently capture subscale features such as Brownian motion of molecules in liquids. As mentioned in [Stam 1999; Bao et al. 2010; Shiny et al. 2010] these sub-scale features can be effectively approximated by a Laplacian-based diffusion. Similarly to salt diffusion presented by Monaghan[Monaghan 2005] the $k$-th concentration is diffused due to mass conservation and Fick's law of material diffusion as

$$
\begin{aligned}
\frac{d C^{k}}{d t} & =\frac{D^{k}}{\rho} \nabla^{2} C^{k}, \\
\left\langle\frac{d C_{i}^{k}}{d t}\right\rangle & =\frac{D^{k}}{\rho_{i}} \sum_{j} V_{j}\left(C_{j}^{k}-C_{i}^{k}\right) \nabla^{2} W\left(\left\|\mathbf{r}_{i}-\mathbf{r}_{j}\right\|, h\right),
\end{aligned}
$$


where $D^{k}$ is the coefficient of molecular diffusivity of $k$-th fluid. We use here the Poly6 kernel function W presented in [Müller et al. 2003].

Since eroded material is usually heavier than fluid, thus we increase diffusion directly to the ground with the settling velocity $\mathbf{w}$ related to the gravity and solid-fluid density difference

$$
\mathbf{w}=\frac{9}{2} r_{s}^{2} \frac{\rho_{s}-\rho_{f}}{\mu} \mathbf{g} f(C),
$$

where sediment grains are assumed to be spherical, $\mu$ is viscosity of the fluid, $r_{s}$ is the radius of sediment grain, $\rho_{s}$ is density of the solid grain, $\rho_{f}$ is the density of the fluid, $\mathbf{g}$ is gravity acceleration vector and $C$ is the concentration of the dissolved material. Since sediment settles down slower with higher concentrations a hindered settling function defined by Richardson-Zaki relation is used

$$
f(C)=1-\left(\frac{C}{C_{\max }}\right)^{e}
$$

where $C_{\max }$ is the maximum allowed concentration of dissolved sediment and exponent is usually $4<e<5.5$ [Wojtan et al. 2007]. The dissolved material is convected by the flow, therefore total velocity $\mathbf{u}=\mathbf{v}+\mathbf{w}$ of the sediment is sum of the fluid velocity $\mathbf{v}$ and the settling velocity $\mathbf{w}$. By observing the Eulerian expression of the diffusion equation

$$
\frac{\partial C}{\partial t}+\mathbf{u} \cdot \nabla C=\frac{\partial C}{\partial t}+(\mathbf{v}+\mathbf{w}) \cdot \nabla C=\frac{D}{\rho} \nabla^{2} C
$$

and the material derivative

$$
d C / d t=\partial C / \partial t+\mathbf{v} \cdot \nabla C .
$$

We get the Lagrangian version of the diffusion equation with explicit advection along the settling velocity as in [Monaghan 2005]

$$
\frac{d C}{d t}=-\mathbf{w} \cdot \nabla C+\frac{D}{\rho} \nabla^{2} C,
$$

where $D$ is the molecular diffusivity. The sedimentation process on the solid-fluid boundary is governed by the settling velocity part of the diffusion equation.

It is important to describe how to implement the advection term $(-\mathbf{w} \cdot \nabla C)$ of diffusion equation along settling velocity $\mathbf{w}$ within SPH. For $i$-th particle having settling velocity $\mathbf{w}_{i}$ and concentration $C_{i}$ advection term is discretized as (similarly to [Krištof et al. 2009])

$$
-\mathbf{w}_{i} \cdot \nabla C_{i}=-\sum_{j}\left\{\begin{array}{ll}
V_{j} C_{j} \mathbf{w}_{i} \cdot \nabla W\left(\left\|\mathbf{p}_{i}-\mathbf{p}_{j}\right\|\right) & \text { case }_{1} \\
V_{i} C_{i} \mathbf{w}_{i} \cdot \nabla W\left(\left\|\mathbf{p}_{i}-\mathbf{p}_{j}\right\|\right) & \text { case }_{2} \\
0 & \text { otherwise }
\end{array},\right.
$$

where summation have the following two cases: case $_{1}=\left(\mathbf{p}_{i}-\mathbf{p}_{j}\right) \cdot \mathbf{w}_{i} \geq 0 \wedge C_{i}<C_{\max }$ and case $_{2}=\left(\mathbf{p}_{i}-\mathbf{p}_{j}\right) \cdot \mathbf{w}_{i}<0 \wedge C_{j}<C_{\max }$.

In contrast to the discretization given in [Krištof et al. 2009] we also model saturation taking into account the maximal allowed concentration $C_{\max }$. In the first case $\left(\left(\mathbf{p}_{i}-\mathbf{p}_{j}\right)\right.$. $\left.\mathbf{w}_{i} \geq 0 \wedge C_{i}<C_{\max }\right), i$-th particle is below $j$-th particle w.r.t. the settling velocity $\mathbf{w}$. This means that the sediment will be transported form the $j$-th particle to the $i$-th particle. In case the $i$-th particle is not saturated yet $\left(C_{i}<C_{\max }\right)$ the $j$-th particle must contribute within the 


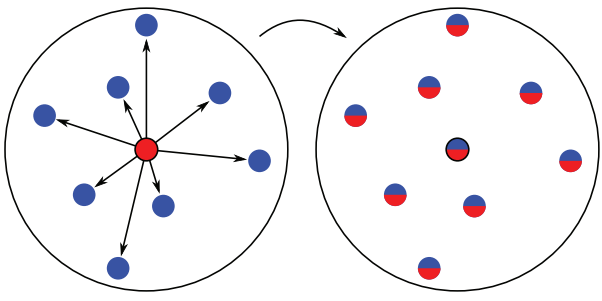

Diffusion

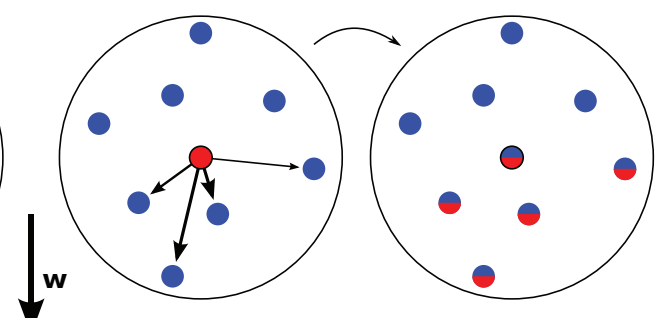

Settling advection

Fig. 6. Left: Diffusion of a particle into its neighbors. Right: Description of advection with respect to the settling velocity w. Material is transported from the particle only downward. Amount of material is depicted with the thickness of the arrows.

SPH summation into the advection term of the $i$-th particle. Since mass must be conserved, the concentration increase on the $i$-particle must result in an equal concentration decrease on the $j$-particle (the $j$-particle has given material to the $i$-th particle). This perfectly matches the second case $\left(\left(\mathbf{p}_{i}-\mathbf{p}_{j}\right) \cdot \mathbf{w}_{i}<0 \wedge C_{j}<C_{\max }\right)$ when indices get reversed.
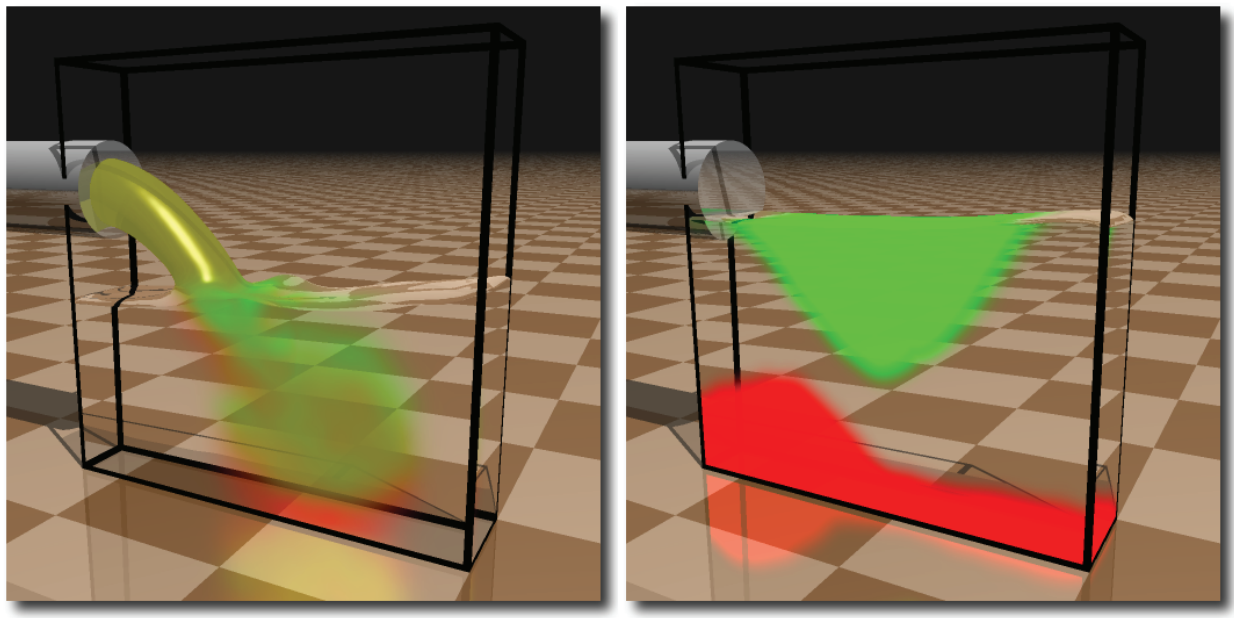

Fig. 7. Separation of two mixed fluid using sedimentation.

\section{DISCUSSION AND RESULTS}

In Figure 1, two and four immiscible fluids are mixed. A clear interface produced by our composite implicit function (Equation 6) can be seen. To convince the reader we have set up different scenes with up to 14 different liquids having various materials, see Figure 1(left) and Figure 8 for detail.We have tested our composite isosurface using three single phase isofunctions [Müller et al. 2003; Onderik et al. 2011; Yu and Turk 2010] reconstructing level set at various thresholds. Higher values provide flatter and less blobby results. However, as shown in Figure 8 (left) reconstructing level set $\varphi(\mathbf{p})=0.75$ (isovalues are normalized to [0,1]) with isofunction proposed in [Müller et al. 2003] thin regions 

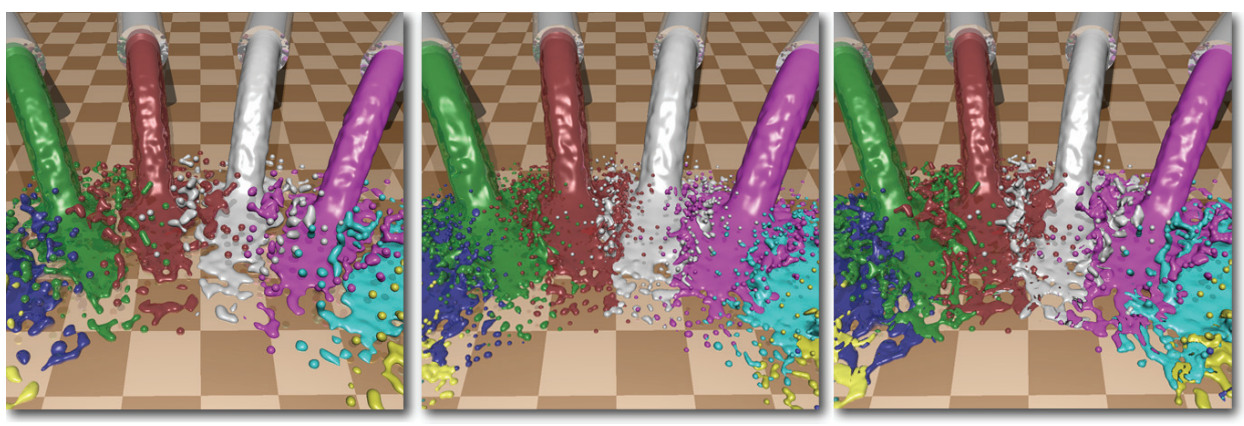

Fig. 8. Mixing of 14 immiscible fluids. Different surface reconstruction methods are compared. Left: Approach from [Müller et al. 2003]. Middle: Approach from [Yu and Turk 2010]. Right: Approach from [Onderik et al. 2011] without refinement.

of fluids near interfaces are discarded, resulting in unnatural loss of fluid volume. This is due to the stronger decay of the kernel function when evaluating the color function. Using our density normalized distance based approach these regions are nicely preserved, see Figure 8 (right). We observed that the approach from [Yu and Turk 2010] (Figure 8 middle) does not preform well in this scene. In regions where several fluid mix, the sampling of the single fluids is low and thus the PCA-based approach gives inaccurate results.

We have set up four testing simulations to demonstrate results of mixing and sedimentation of miscible particle based fluids. All scene were rendered using our volume ray tracing system. Since we did not implement unbiased physically correct rendering, the following strategy has been taken to account with material concentrations.

During the ray traversal through the liquid, concentrations of dissolved materials are sampled in regular distances along the ray. We use the SPH interpolation of concentrations from neighbor particles to get concentration for the investigated position. Between two immiscible interfaces, we track the maximum concentration of any fluid and use this maximum for alpha-blending between the color given by the refracted ray and the color of the dissolved media. We have used an additive approach for color mixing. Final color of the media is a concentration based average of the initial material colors as described in Equation 9.

In Figure 7, the sedimentation process is illustrated. Mixture of a red and green fluid are poured into an aquarium. Using sedimentation part of the diffusion equation (Equation 16), the red fluid is separated from the green liquid. Diffusion process is illustrated in the Figure 9, where two different fluids are poured into an aquarium. On the top, the molecular diffusivity $D$ from Equation 15 is set to 0 . The two fluids do not diffuse together and with the water in the aquarium. On the bottom, $D=1$. The fluids diffuse together and create a purple mixture that is dissolved in the water. It is known that discrete Laplacian is unstable when integrated explicitly. In all our examples $D<=1$, and we did not observe any stability issues with the time step $\Delta t=0.001 \mathrm{~s}$. For a higher $D$ smaller times are needed, but such a large diffusivity is rarely needed. A dam break scene with two miscoible fluids is depicted in Figure 2. The yellow fluid has a higher viscosity than the transparent.

A sequence of sedimenting fluids is shown in Figure 10. Molecular diffusivity is set to 0 
thus the fluids only sediment and do not diffuse. First, the purple and green fluid sediment to the bottom of the aquarium. Afterwards a black fluid is poured into the aquarium having a non-zero downward directing settling velocity and zero molecular diffusion. Therefore it sediments to the bottom without getting mixed with the other fluids.

\section{CONCLUSION AND FUTURE WORK}

To conclude, we have proposed an approach for particle-based simulation that can handle arbitrary number of miscible as well as immiscible fluids. In order to track interfaces between immiscible fluids, we devised a novel composite implicit function. To simulate miscible fluids we tracked concentrations of dissolved material. To advect the concentration a Laplacian-based diffusion was used. We improved the method by adding a directiondependent diffusion enabling us to simulate sedimentation of the dissolved material.

In the future we would like to parallelize the whole algorithm and exploit the power of GPU. But we think that this should be a straightforward task.

\section{Acknowledgment}

This research was partially supported by Comenius University grant for young researches.

\section{REFERENCES}

BAO, K., Wu, X., Zhang, H., AND Wu, E. 2010. Volume fraction based miscible and immiscible fluid animation. Comput. Animat. Virtual Worlds 21, 401-410.

BATCHELOR, G. 1967. An introduction to fluid mechanics.

Carlson, M., Mucha, P. J., Van Horn, III, R. B., And Turk, G. 2002. Melting and flowing. In Proceedings of the 2002 ACM SIGGRAPH/Eurographics symposium on Computer animation. SCA '02. ACM, New York, NY, USA, 167-174.

FEDKIW, R., StAm, J., AND JENSEN, H. W. 2001. Visual simulation of smoke. In SIGGRAPH '01: Proceedings of the 28th annual conference on Computer graphics and interactive techniques. ACM, New York, NY, USA, $15-22$.

Harris, M. J., Baxter, W. V., Scheuermann, T., And Lastra, A. 2003. Simulation of cloud dynamics on graphics hardware. In HWWS '03: Proceedings of the ACM SIGGRAPH/EUROGRAPHICS conference on Graphics hardware. Eurographics Association, Aire-la-Ville, Switzerland, Switzerland, 92-101.

KAng, N., PARK, J., Noh, J., AND ShIn, S. Y. 2010. A hybrid approach to multiple fluid simulation using volume fractions. Computer Graphics Forum 29, 2, 685-694.

Keiser, R., Adams, B., Gasser, D., Bazzi, P., Dutre, P., And Gross, M. 2005. A unified lagrangian approach to solid-fluid animation. In Symposium on Point-Based Graphics, M. Gross, H. Pfister, M. Alexa, and S. Rusinkiewicz, Eds. Eurographics Association, Zurich, Switzerland, 125-133.

KIM, B. 2010. Multi-phase fluid simulations using regional level sets. ACM Trans. Graph. 29, 175:1-175:8.

KRIŠToF, P., BEnEŠ, B., KřIVÁneK, J., AND ŠŤAVA, O. 2009. Hydraulic erosion using smoothed particle hydrodynamics. Computer Graphics Forum (Proceedings of Eurographics 2009) 28, 2.

Lenaerts, T., AdAms, B., And Dutré, P. 2008. Porous flow in particle-based fluid simulations. In $S I G$ GRAPH '08: ACM SIGGRAPH 2008 papers. ACM, New York, NY, USA, 1-8.

Losasso, F., Irving, G., And Guendelman, E. 2006. Melting and burning solids into liquids and gases. IEEE Transactions on Visualization and Computer Graphics 12, 3, 343-352. Member-Ron Fedkiw.

Losasso, F., Shinar, T., Selle, A., And Fedkiw, R. 2006. Multiple interacting liquids. In SIGGRAPH '06: ACM SIGGRAPH 2006 Papers. ACM Press, New York, NY, USA, 812-819.

Monaghan, J. 2005. Smoothed particle hydrodynamics. Reports on Progress in Physics 68, 1703-1759. 


\title{
J. ONDERIK, M. CHLÁDEK, R. ĎURIKOVIČ
}

Müller, M., Charypar, D., And Gross, M. 2003. Particle-based fluid simulation for interactive applications. In SCA '03: Proceedings of the 2003 ACM SIGGRAPH/Eurographics symposium on Computer animation. Eurographics Association, Aire-la-Ville, Switzerland, Switzerland, 154-159.

Müller, M., Solenthaler, B., Keiser, R., And Gross, M. 2005. Particle-based fluid-fluid interaction. In SCA '05: Proceedings of the 2005 ACM SIGGRAPH/Eurographics symposium on Computer animation. ACM Press, New York, NY, USA, 237-244.

Onderik, J., ChládeK, M., AND Ďurikovič, R. 2011. Sph with small scale details and improved surface reconstruction. In SCCG '11: Proceedings of the Spring Conference on Computer graphics. ACM Press, Viničné, Slovakia.

PARK, J., KIM, Y., Wi, D., KANG, N., ShIN, S. Y., AND YONG NOH, J. 2008. A unified handling of immiscible and miscible fluids. Journal of Visualization and Computer Animation 19, 455-467.

Rasmussen, N., Nguyen, D. Q., Geiger, W., And Fedkiw, R. 2003. Smoke simulation for large scale phenomena. ACM Trans. Graph. 22, 3, 703-707.

Runguiratananon, W., Szego, Z., KAnAmori, Y., And Nishita, T. 2008. Real-time animation of sandwater interaction. In PG '08: Proceedings od the Pacific Graphics 2008.

ShinY, S.-H., KAMZ, H. R., AND KIMX, C.-H. 2010. Hybrid simulation of miscible mixing with viscous fingering. Computer Graphics Forum 29, 2, 675-683.

Solenthaler, B., Schläfl, J., And PAJARola, R. 2007. A unified particle model for fluid-solid interactions. Computer Animation and Virtual Worlds 18, 69-82.

StAM, J. 1999. Stable fluids. In SIGGRAPH '99: Proceedings of the 26th annual conference on Computer graphics and interactive techniques. ACM Press/Addison-Wesley Publishing Co., New York, NY, USA, 121128.

Wojtan, C., Carlson, M., Mucha, P. J., And Turk, G. 2007. Animating corrosion and erosion. In Eurographics Workshop on Natural Phenomena.

YU, J. AND TURK, G. 2010. Reconstructing surfaces of particle-based fluids using anisotropic kernels. In Proceedings of the 2010 ACM SIGGRAPH/Eurographics Symposium on Computer Animation. SCA '10. Eurographics Association, Aire-la-Ville, Switzerland, Switzerland, 217-225.

ZHU, Y. AND BRIDson, R. 2005. Animating sand as a fluid. ACM Trans. Graph. 24, 965-972.

\author{
Authors' addresses: \\ Juraj Onderik \\ Mathematics, Physics and Informatics, \\ Comenius University, 84248 Bratislava, Slovak Republic \\ http: //www.fmph.uniba.sk \\ email: juraj.onderik@fmph.uniba.sk \\ Michal Chládek \\ Mathematics, Physics and Informatics, \\ Comenius University, 84248 Bratislava, Slovak Republic \\ http: //www.fmph.uniba.sk \\ email: michal.chladek@fmph.uniba.sk \\ Roman Ďurikovič \\ Faculty of Mathematics, Physics and Informatics, \\ Comenius University, \\ 84248 Bratislava, Slovak Republic; \\ http://www.sccg.sk/ durikovic \\ email: roman.durikovic@fmph.uniba.sk
}


ANIMA MULTIPLE INTERACTING MISCIBLE AND IMMISCIBLE FLUIDS ...
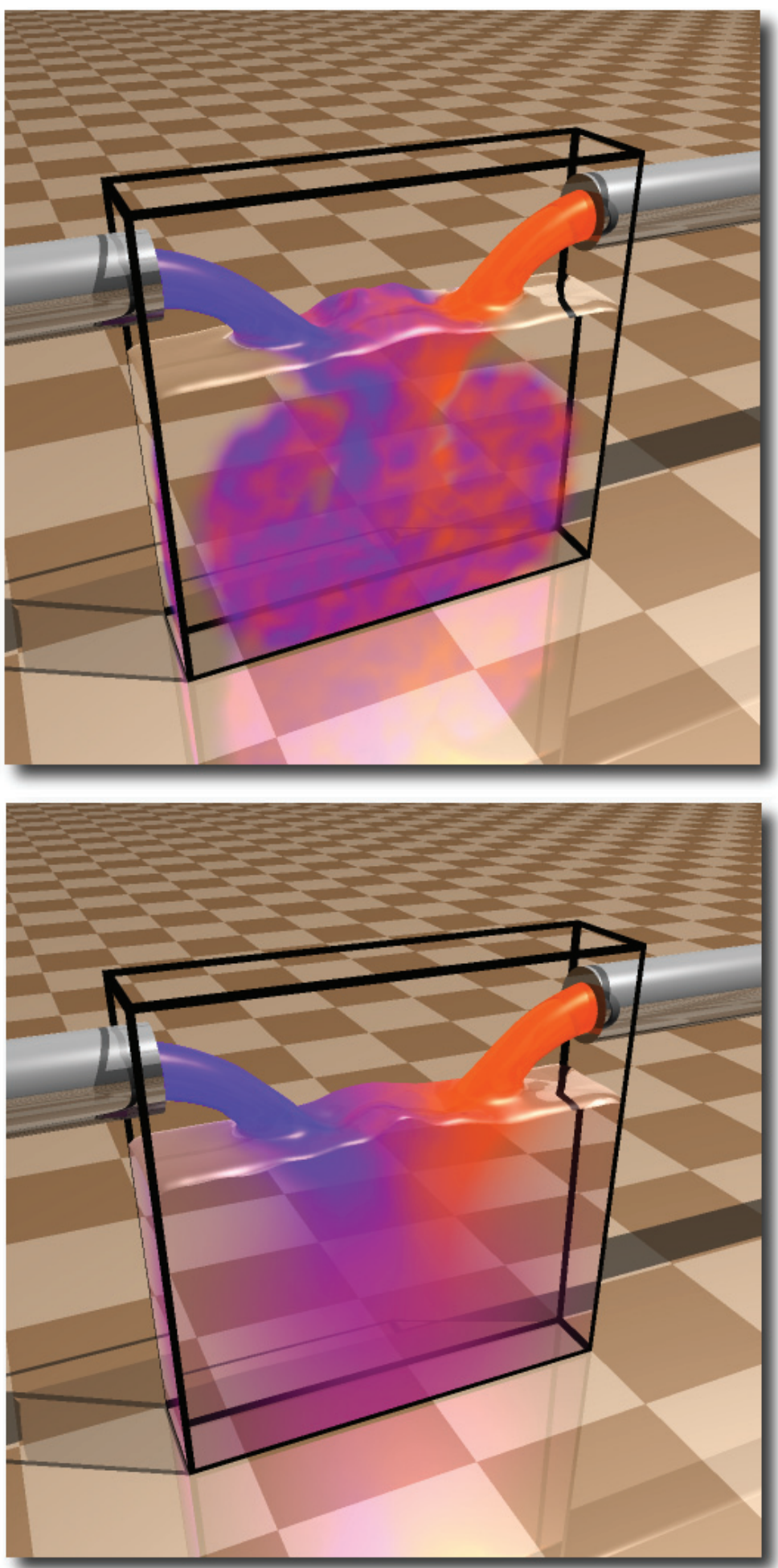

Fig. 9. Mixing miscible fluids using the molecular diffusivity $D=0$ (top) and $D=1$ (bottom). 

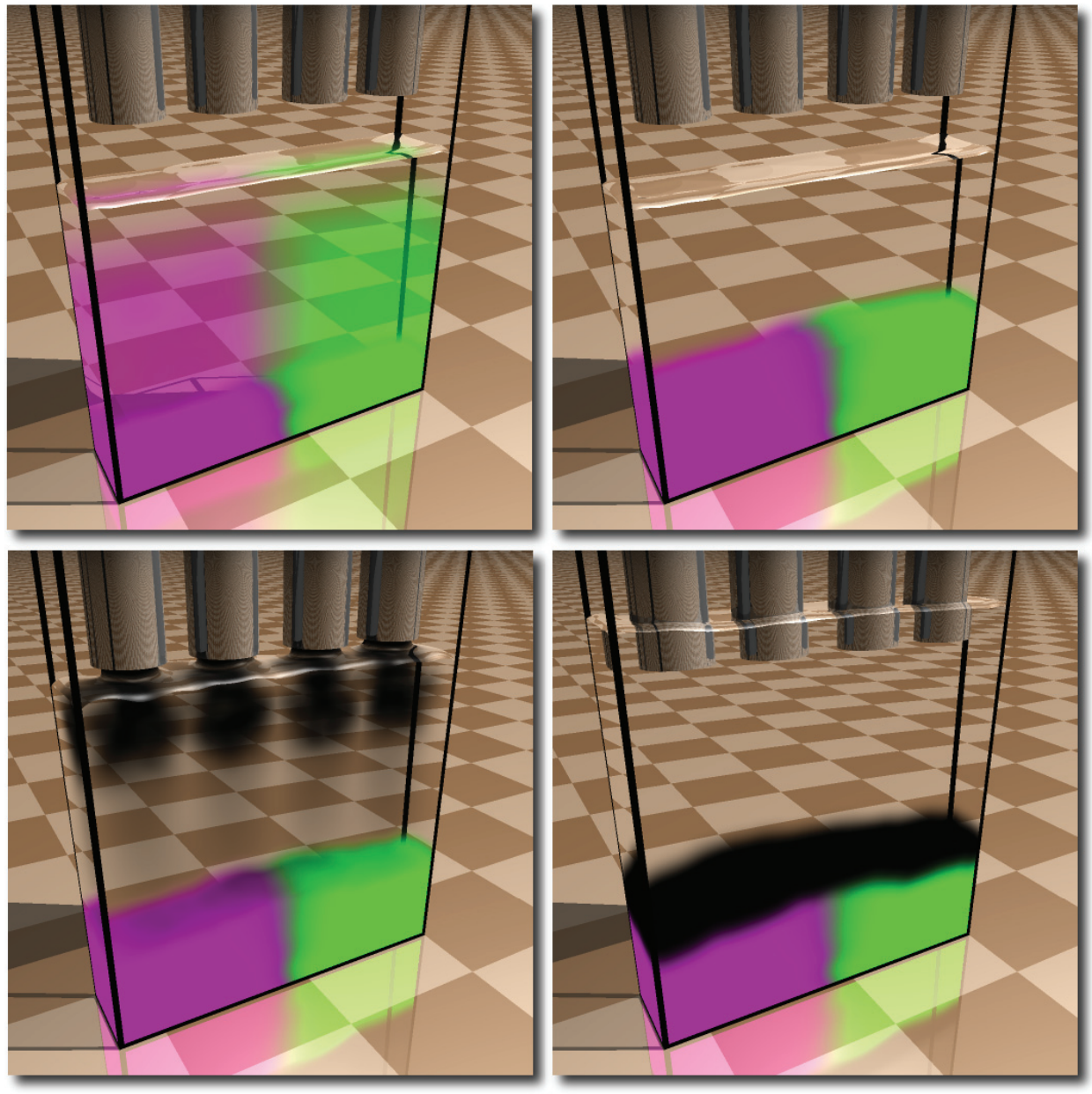

Fig. 10. Sequence of sedimenting fluids in aquarium. First, the green and purple fluids sediment to the bottom(first row). Afterwards a black fluid is poured and sediments to the bottom as well. 